\title{
Color Fourier Descriptor for Defect Image Retrieval
}

\author{
Iivari Kunttu ${ }^{1}$, Leena Lepistö ${ }^{1}$, Juhani Rauhamaa ${ }^{2}$, and Ari Visa ${ }^{1}$ \\ ${ }^{1}$ Tampere University of Technology, Institute of Signal Processing \\ P.O. Box 553, FI-33101 Tampere, Finland \\ \{Iivari.Kunttu, Leena.Lepisto,Ari.Visa\}@tut.fi \\ http: //www.tut.fi \\ ${ }^{2}$ ABB Oy, Process Industry P.O. Box 94, FI-00381 Helsinki, Finland \\ Juhani.Rauhamaa@fi.abb.com \\ http: //www.abb.com
}

\begin{abstract}
The shapes of the objects in the images are important in the contentbased image retrieval systems. In the contour-based shape description, Fourier descriptors have been proved to be effective and efficient methods. However, in addition to contour shape, Fourier description can be used to characterize also the color of the object. In this paper, we introduce new Color Fourier descriptors. In these descriptors, the boundary information is combined with the color of the object. The results obtained from the retrieval experiments show that by combining the color information with the boundary shape of the object, the retrieval accuracy can be clearly improved. This can be done without increasing the dimensionality of the descriptor.
\end{abstract}

\section{Introduction}

Nowadays, the problem of image retrieval plays a remarkable role in the fields of image analysis and pattern recognition. With increasing amount of real-world image data to be processed and stored, the development of powerful retrieval tools has become an essential subject of research work. The description of the objects occurring in the images is based on visual features extracted from them. In addition to color and texture, shape is one of the most important features used to characterize the objects occurring in the images as accurately as possible. These features are widely used in content-based image retrieval systems [1],[8].

On the other hand, classification accuracy (effectiveness) of a certain descriptor is not an adequate measure for its usefulness in the retrieval. Due to the increasing number of online retrieval solutions, computational efficiency is nowadays considered equally important as effectiveness [10]. In retrieval applications, the matter of computational complexity is twofold, namely the cost of image database indexing and retrieval. In the indexing, the features (descriptors) are extracted from the database images. Although this part is not always online operation, the feature extraction should not be a computationally heavy. More importantly, retrieval is always performed in real time. Therefore, the descriptors used in retrieval are required to be compact. The compactness of a de- 
scriptor depends on its dimensionality, because the retrieval time is proportional to the descriptor dimensions. Consequently, low-dimensional descriptors are preferred.

In this paper, we concentrate on object description that is based on Fourier transform. Fourier-based methods are widely used in shape description [6]. Fourier descriptors have been found to be accurate in shape classification in several comparisons, [2],[3],[4],[9]. In addition to good retrieval and classification accuracy, there are also other reasons which make Fourier descriptors popular among the contour-based shape representations. The main advantages of the Fourier-based shape representations are that they are compact and computationally light methods. Furthermore, they are easy to normalize and their matching is very simple. Also the sensitivity to noise is low, when only low frequency Fourier coefficients are used as descriptors.

In addition to object shape, its color is often equally important feature. In retrieval systems, colors are usually characterized using relatively high-dimensional descriptors, like histograms [1] or other statistical measures. On the other hand, the number of descriptors that efficiently combine color and shape is very small. In the work of Mehtre et al., [5] color and shape of the object were combined. This approach, however, was based on quite complicated clustering method. Furthermore, the approach used moment-based shape features that are computationally more expensive than for example Fourier descriptors.

In this paper, we present a new approach to the use of Fourier descriptors in the characterization of image content. Hence, we show that the Fourier descriptor is capable of describing also other features of the object than its contour. In our approach, we add the object color to the Fourier-based contour description. In this way, the obtained descriptor is able to more accurate object description in the retrieval process. However, the color information does not increase the dimensionality of the obtained descriptor.

The organization of this paper is the following. In section two, the principles of Fourier descriptors and our approach, Color Area Fourier, are presented. Section three reports the retrieval experiments carried out using real industrial defect images. The proposed method is discussed in section four.

\section{Object Description}

In this section, the common methods for shape description using Fourier-based methods are presented. In addition to that, our approach to combine object color information with its contour in Fourier description is presented.

\subsection{Fourier Descriptors}

Shape signatures. Shape signature is a 1D boundary function $f(k)$ that represents the boundary of a 2D object. The functions are either real-valued or complex. Complex coordinate function [2] is the simplest and best-known boundary presentation. It presents the coordinates of the boundary $\left(x_{k}, y_{k}\right)$ in an object centered complex coordinate system: 


$$
z(k)=\left(x_{k}-x_{c}\right)+j\left(y_{k}-y_{c}\right)
$$

for $k=0,1,2, \ldots, N-1$, in which $N$ is the length of the boundary and $\left(x_{c}, y_{c}\right)$ is the centroid of the object. Area function [11] is an example of real-valued shape signatures and it is defined as the area of the triangle formed by two boundary points and centroid in the object centered coordinate system:

$$
a(k)=\frac{\left|\left(x_{k}-x_{c}\right)\left(y_{k+1}-y_{c}\right)-\left(x_{k+1}-x_{c}\right)\left(y_{k}-y_{c}\right)\right|}{2}
$$

Hence, both signatures represent the boundary independent of the location of the object in the image.

Signatures for color and shape. The object signatures of the proposed descriptors use the same basic approach as the complex-valued shape signatures of equation (1). Hence, by combining two real-valued 1D signals it is possible to form a 1D complex signal. In this paper, we combine the object color to its boundary information. This is made by combining the color of the object region defined by the shape signature with the signature itself. The color value $C_{k}$ can be e.g. the mean of the selected color component at each object region $k$. In the case of area function, the region corresponds to the image pixels covered by the area of the triangle defined by equation (2). The signature for color and shape of an object is expressed as complex numbers:

$$
c_{a}(k)=a_{k}+j C_{k}
$$

Hence the signature of equation (3) combines the real-valued boundary information with object color distribution.

Fourier description. The descriptor based on a signature function can be formed in several ways. Fourier transform is a commonly used method for this purpose. Fourier transformation of a boundary function generates a set of complex numbers which are called Fourier descriptors. Fourier descriptors characterize the object shape in a frequency domain. The discrete Fourier transform for a boundary function $f(k)$ is:

$$
F_{n}=\frac{1}{N} \sum_{k=0}^{N-1} f(k) e^{-j 2 \pi n k / N}
$$

for $n=0,1,2, \ldots, N-1$. The general shape of the object is represented by the lower frequency descriptors, whereas high frequency descriptors represent the fine details of the object shape. The descriptors have to be normalized to achieve invariance to translation, rotation, and scaling. Translation invariance of is based on the object centered shape signatures. The descriptors can be made rotation invariant by ignoring the phase information and using only the magnitudes of the transform coefficients $\left|F_{n}\right|$. The scale can be normalized by dividing the magnitudes by $\left|F_{0}\right|$ or $\left|F_{1}\right|$, depending on the shape representation method [2]. 
Feature vectors. A common approach to object description is to use only a subset of low frequency coefficients that carry the most of the object information. This way the shape can be effectively presented using a relatively short feature vector. For complex-valued shape signatures, the coefficients are taken from positive and negative frequency axis:

$$
x=\left[\frac{\left|F_{-(L / 2-1)}\right|}{\left|F_{1}\right|}, \cdots, \frac{\left|F_{-1}\right|}{\left|F_{1}\right|}, \frac{\left|F_{2}\right|}{\left|F_{1}\right|}, \cdots, \frac{\left|F_{L / 2}\right|}{\left|F_{1}\right|}\right]^{T}
$$

in which $L$ is a constant value that defines the dimensionality of the feature vector. When this description is formed for the transform coefficients obtained from complex coordinate function of equation (1), it is called Contour Fourier method [2]. In this paper, this kind of feature vector is applied also to Color Area Fourier descriptor that uses complex-valued signature of equation (3). However, in the case of Color Area Fourier descriptor, the normalization is carried out using $\left|F_{0}\right|$ instead of $\left|F_{1}\right|$ :

$$
x=\left[\frac{\left|F_{-(L / 2)}\right|}{\left|F_{0}\right|}, \cdots, \frac{\left|F_{-1}\right|}{\left|F_{0}\right|}, \frac{\left|F_{1}\right|}{\left|F_{0}\right|}, \cdots, \frac{\left|F_{L / 2}\right|}{\left|F_{0}\right|}\right]^{T}
$$

The difference between the feature vectors can be explained by differences between the signatures. In the case of Contour Fourier method of equation (5), the signature uses merely boundary information that is represented in location-independent manner. Therefore, scale is normalized using the first non-zero coefficient, $\left|F_{1}\right|$. On the other hand, Color Area Fourier descriptor uses complex-valued signature of equation (3) in which contour shape is represented by centroid distance. Therefore, the mean value of the signature function differs from zero. This causes the normalization by $\left|F_{0}\right|$, which is the transform coefficient representing the mean value of the signal.

The real-valued shape representation, Area Fourier [2] uses the area function as shape signature. Because this signature is real, only half of the transform coefficients are needed to characterize the shape [2]:

$$
x=\left[\frac{\left|F_{1}\right|}{\left|F_{0}\right|}, \frac{\left|F_{2}\right|}{\left|F_{0}\right|}, \cdots, \frac{\left|F_{L}\right|}{\left|F_{0}\right|}\right]^{T}
$$

Also with this descriptor type, the normalization is carried out using the mean component, $\left|F_{0}\right|$, to remove the effect of the mean of the area function.

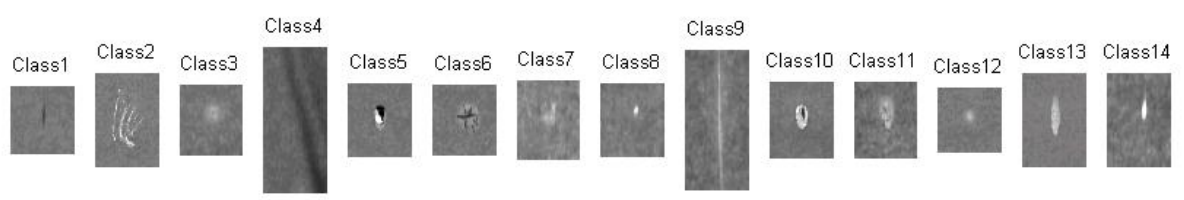

Fig. 1. An example image of each paper defect class in the testing database 


\section{Retrieval Experiments}

In this section, we present the retrieval experiments carried out using a real defect image database. We compare the retrieval performance of the proposed Color Area Fourier approach to the ordinary Fourier shape description methods, which describe only the boundary line of the object.

\subsection{Defect Image Database}

For testing purposes, we used paper defect images that were collected from an industrial process. The images were taken from the paper manufacturing process using a paper inspection system [7] that produces gray level images of the defects. The reason for the collection of the defect image databases in the process industry is the practical need of controlling the quality and production [7]. In industrial imaging solutions, there is a need to retrieve the defect images from the databases. In these images, the defect shape and gray level are the most essential features that describe the defect type. Therefore, effective methods for the shape and gray level representation are needed in the retrieval and classification of the defect images. The defects occurring in the paper can be for example holes, wrinkles or different kinds of dirt spots. The test set consisted of 1204 paper defects, which represented 14 defect classes so that each class consisted of 27-103 images. An example image of each paper defect class is presented in figure 2. Within the classes of the defect database, there were differences in the gray levels, size, and orientation of the defects.
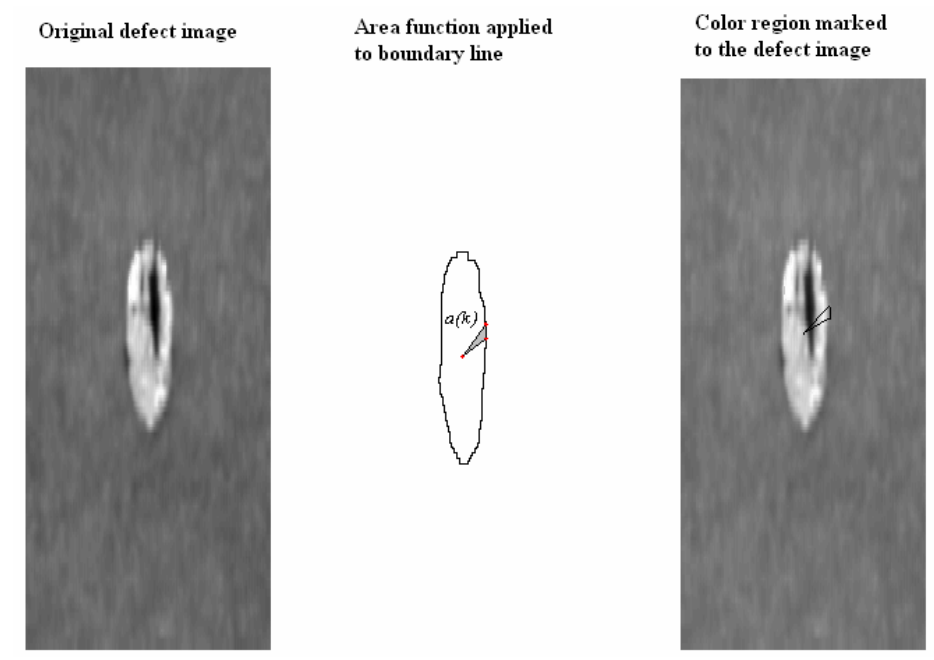

Fig. 2. The principle of Color Area Fourier descriptor applied to a paper defect image 


\subsection{Retrieval}

In this paper, we compare our Color Area Fourier approach to Area Fourier method and Contour Fourier method that have been proved to be effective Fourier-based shape descriptors in retrieval of defect shapes [3].

The Fourier-based shape descriptors (Area Fourier and Contour Fourier) were calculated based on the defect contours. The descriptors used triangular color regions that were defined from the defect images so that the triangle was drawn between object centroid and two consequent boundary points (figure 2). The feature vectors of the descriptors were formed using equations (7) and (5), respectively. In the case of Color Area Fourier descriptor, the color information was added to the area-based shape description. The defects are presented as gray level images, which means that only the intensity component was used to represent the color. The color value $C_{k}$ was selected to be the mean gray level of the triangular region. The descriptors were formed using feature vector of equation (6).

In the comparison, low-dimensional descriptors were preferred, and hence we used two lengths of the vectors $(L)$, namely 8 and 16 . In the retrieval experiments, the distance measure between the feature vectors was selected to be the Euclidean distance. The retrieval experiments were made using leaving one out method. In this method, each image in turn is left out from the test set and used as a query image; whereas the other images in the test set form a testing database. The performance of the retrieval was measured by calculating a precision versus recall curve for each query.

The average precision/recall curves for the database are presented in figure 3 . The results show that the Fourier descriptors combined with object color (Color Area Fourier) outperform clearly the Area Fourier descriptor. On the other hand, the
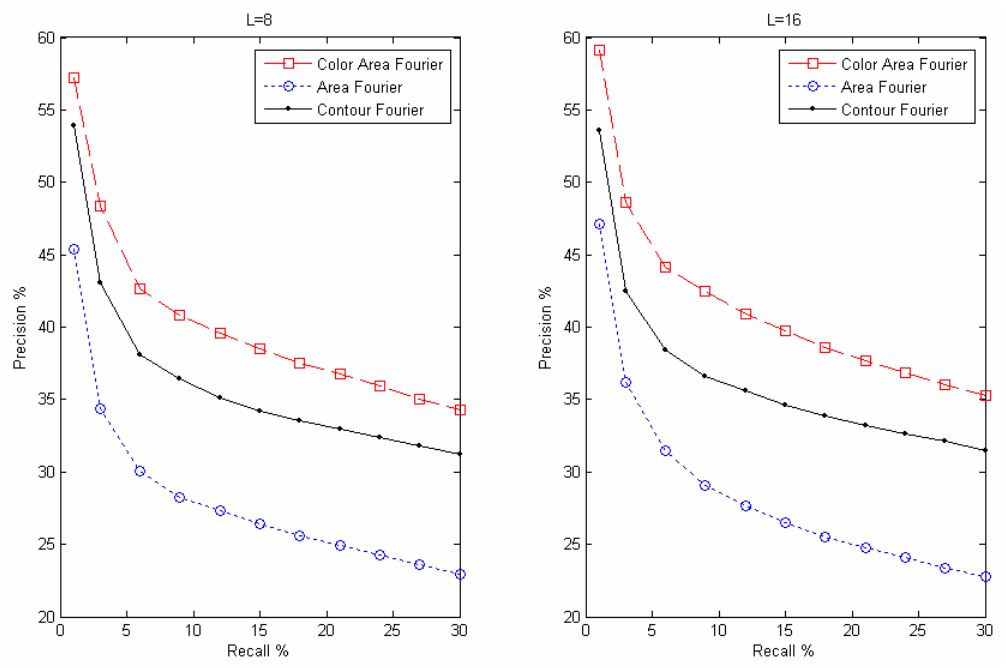

Fig. 3. The average precision/recall curves of the retrieval experiments calculated for each descriptor type 
proposed descriptors outperform also Contour Fourier method that is the most accurate shape-based Fourier descriptor in defect image retrieval [3]. It is essential to note that this improvement does not increase dimensionality of the feature vectors, when Color Area Fourier and Contour Fourier descriptors are compared. Furthermore, when the same distance metrics is applied, the computational cost of retrieval is equal with the proposed descriptors and the conventional Fourier shape descriptors.

\section{Discussion}

In this paper, a new approach to Fourier-based object presentation was introduced. Our approach, Color Area Fourier descriptor, combines the color and shape information of an object into a single feature vector. The obtained vector is as low dimensional and easy to match as any other shape-based Fourier descriptor. However, our experiments showed that Color Area Fourier descriptor outperforms the other Fourier-based shape descriptors in terms of retrieval accuracy.

In the experiments, a database of complex shapes was used. The shapes in the database represent defects that occur in an industrial process. Self-evidently, the accuracy of the descriptors is the most important criterion also in this retrieval problem. On the other hand, the matter of computational efficiency is essential in this case, like in the most of the real-world image retrieval tasks. Therefore, compact features are required. The experiments showed that combining the color information to a Fourier descriptor, additional retrieval accuracy can be achieved without increasing computational cost of retrieval. Therefore, the Color Area Fourier descriptor presented in this paper is an effective and efficient tool for describing complex objects in image retrieval and classification.

\section{References}

1. Del Bimbo, A.: Visual Information Retrieval, Morgan Kaufmann Publishers, San Fransisco, (2001)

2. Kauppinen, H., Seppänen, T., Pietikäinen, M.: An Experimental Comparison of Autoregressive and Fourier-Based Descriptors in 2D Shape Classification, IEEE Transactions on Pattern Analysis and Machine Intelligence, Vol. 17, No. 2 (1995) 201-207

3. Kunttu, I., Lepistö, L., Rauhamaa, J., Visa, A.: Multiscale Fourier Descriptor for ShapeBased Image Retrieval, Proceedings of $17^{\text {th }}$ International Conference on Pattern Recognition, Cambridge, UK, Vol. 2 (2004) 765-768

4. Mehtre, B.M., Kankanhalli, M.S., Lee, W.F.: Shape Measures for Content Based Image Retrieval: A Comparison, Information Processing Management, Vol. 33, No 3, (1997) 319-337

5. Mehtre, B.M., Kankanhalli, M.S., Lee, W.F.: Content-Based Image Retrieval Using a Composite Color-Shape Approach. Information Processing \& Management Vol. 34, No. 1, (1998) 109-120.

6. Persoon, E., Fu, K.: Shape Discrimination Using Fourier Descriptors, IEEE Transactions on Systems, Man, and Cybernetics, Vol. 7 (1977) 170-179 
7. Rauhamaa, J., Reinius, R.: Paper Web Imaging with Advanced Defect Classification, Proceedings of the 2002 TAPPI Technology Summit, Atlanta, Georgia (2002)

8. Smeulders, A. W. M., Worring, M., Santini, S., Gupta, A., Jain, R.: Content-Based image Retrieval at the End of the Early Years. IEEE Transactions Pattern Analysis and Machine Intelligence Vol. 22, No. 12 (2000) 1349-1380.

9. Zhang, D., Lu, G.: A Comparative Study of Curvature Scale Space and Fourier Descriptors for Shape-Based image Retrieval, Journal of Visual Communication and Image Representation, Vol. 14, No. 1 (2003) 41-60

10. Zhang, D., Lu, G.: Review of shape representation and description techniques. Pattern Recognition, Vol. 37, No. 1, (2004) 1-19

11. Zhang, D.S., Lu, G.: Study and evaluation of different Fourier methods for image retrieval. Image and Vision Computing Vol. 23, (2005) 33-49 